\title{
MARKOVIAN PROCESSES ON MUTUALLY COMMUTING VON NEUMANN ALGEBRAS
}

\author{
CARLO CECCHINI \\ Dipartimento di Matematica e Informatica, Università di Udine \\ Via delle Scienze 206 (loc. Rizzi), 33100 Udine, Italy \\ E-mail: cecchini@dimi.uniud.it
}

1. The aim of this paper is to study markovianity for states on von Neumann algebras generated by the union of (not necessarily commutative) von Neumann subagebras which commute with each other. This study has been already begun in [2] using several a priori different notions of noncommutative markovianity. In this paper we assume to deal with the particular case of states which define odd stochastic couplings (as developed in [3]) for all couples of von Neumann algebras involved. In this situation these definitions are equivalent, and in this case it is possible to get the full noncommutative generalization of the basic classical Markov theory results. In particular we get a correspondence theorem, and an explicit structure theorem for Markov states.

2. Let $M$ be a von Neumann algebra acting on an Hilbert space $H$. For $\xi$ in $H$ we denote by $\omega_{\xi}$ the vector state on $B(H)$ implemented by $\xi$. In order to simplify our notations we shall often write $\left(\omega_{\xi}\right)_{M}$ for $\left.\omega_{\xi}\right|_{M}$ or simply $\left(\omega_{\xi}\right)_{\alpha}$ if the von Neumann algebra involved is endowed with an index $\alpha$.

We shall say $C$ is a self-dual positive cone for $M$ in $H$ if there is a separating vector $\Omega$ for $M$ in $H$, such that $C$ is the selfdual positive cone for $E M E$ in $E H$ (in the sense of the modular theory for von Neumann algebras) which contains $\Omega$, with $E$ the orthogonal projection from $M$ to the closure of $\{a \Omega, a \in M\}$.

Let $\gamma$ be an index, $M_{\gamma}$ be a von Neumann algebra acting on a Hilbert space $H$, and let $\Omega$ be a vector in $H$ which is separating for $M_{\gamma}$. We shall denote by $H_{\gamma}$ the closure of $\left\{a \Omega, a\right.$ in $\left.M_{\gamma}\right\}$, by $E_{\gamma}$ the orthogonal projection from $H$ to $H_{\gamma}$, and with the usual notations endow with an index $\gamma$ the objects of the modular theory for the action of

1991 Mathematics Subject Classification: Primary 46L50; Secondary 46L10.

This paper has been written with the support of the Italian M.U.R.S.T funds $40 \%$ and $60 \%$. The paper is in final form and no version of it will be published elsewhere. 
$E_{\gamma} M_{\gamma} E_{\gamma}$ on $H_{\gamma}$; in particular we denote by $J_{\gamma}$ the isometrical involution for $E_{\gamma} M_{\gamma} E_{\gamma}$ on $H_{\gamma}$ which leaves $C_{\gamma}$ invariant. If $M_{\mu}, M_{\nu}$ are von Neumann algebras with $M_{\nu}$ contained in $M_{\mu}$ we shall denote by $\varepsilon_{\mu, \nu}$ the $\omega$ - (or generalized) conditional expectation (cf. [1]) from $M_{\mu}$ to $M_{\nu}$ which preserves $\omega_{\Omega}$. We shall denote by $F\left(\varepsilon_{\mu, \nu}\right)$ the set of the fixed points under $\varepsilon_{\mu, \nu}$. If the $\omega$-conditional expectation is a norm one projection (i.e. $F\left(\varepsilon_{\mu, \nu}\right)=M_{\nu}$ ) we shall say $M_{\nu}$ to be expected in $M_{\mu}$ with respect to $\left(\omega_{\Omega}\right)_{\mu}$.

We shall start by considering two mutually commuting (not necessarily commutative) von Neumann algebras $M_{1}$ and $M_{2}$ (i.e. for all $a_{1}$ in $M_{1}, a_{2}$ in $M_{2}$ we assume $a_{1} a_{2}=a_{2} a_{1}$ ) and assume $\Omega$ in $H$ to be separating for both $M_{1}$ and $M_{2}$.

Let $a_{1}$ be in $M_{1}$. Then $E_{2} a_{1} E_{2}$ commutes with $M_{2}$; so there is a unique operator $\lambda_{1,2}\left(a_{1}\right)$ such that $E_{2} a_{1} \xi=J_{2} \lambda_{1,2}\left(a_{1}^{+}\right) J_{2} \xi$ for all $\xi$ in $H_{2}$. It is immediate to check that the mapping $\lambda_{1,2}: M_{1} \mapsto M_{2}$ is a linear, ultraweakly continuous, completely positive contraction. If $\xi, \eta$ are in $C_{1}$ we have, for all $a_{1}$ in $M_{1}$ :

$$
\begin{aligned}
& \omega_{\eta+i \xi}\left(\lambda_{1,2}\left(a_{1}\right)\right)=\left\langle\eta+i \xi, \lambda_{1,2}\left(a_{1}\right)(\eta+i \xi)\right\rangle \\
& =\left\langle\eta+i \xi, J_{2} E_{2} a_{1}^{+} J_{2}(\eta+i \xi)\right\rangle=\left\langle E_{2} a_{1}^{+}(\eta-i \xi), J_{2}(\eta+i \xi)\right\rangle \\
& =\left\langle E_{2} a_{1}^{+}(\eta-i \xi), \eta-i \xi\right\rangle=\left\langle\eta-i \xi, a_{1}(\eta-i \xi)\right\rangle \\
& =\omega_{\eta-i \xi}\left(a_{1}\right) .
\end{aligned}
$$

This proves that the mapping $\lambda_{1,2}$ is the dual mapping of an odd stochastic transition $\varrho_{2,1}$ from $\left(M_{2}\right)^{*}$ to $\left(M_{1}\right)^{*}$ (cf. [3]). If we define $\varrho_{2,1}$ symmetrically the same proof yields that $\left(\varrho_{2,1}, \varrho_{1,2}\right)$ is an $\Omega$ implemented odd stochastic coupling for $M_{1}$ and $M_{2}$ as defined in [3] provided we assume the following

Condition. Let $\sigma_{i}^{t}$ be the modular authomorphism group on $M_{i}$ for $\left(\omega_{\Omega}\right)_{i}(i=1,2)$. For any $t$ real and $a_{1}$ in $M_{1}$ we have $\lambda_{1,2}\left(\sigma_{1}^{t}\left(a_{1}\right)\right)=\sigma_{2}^{t}\left(\lambda_{1,2}\left(a_{1}\right)\right)$.

In the following we shall always assume, without recalling it explicitly, this intertwining condition to be satisfied for all pairs of mutually commuting algebras we consider with reference to the given vector.

It is proved in [3] that in this situation there is an antiunitary operator $\mathbf{J}$ on the Hilbert space $H_{\{1,2\}}$ spanned by $H_{1} \cup H_{2}$ which commutes with both $E_{1}$ and $E_{2}$ and such that the action of $\mathbf{J} E_{i}$ on $H_{i}$ coincides with the action of $J_{i}(i=1,2)$. This implies immediately the following lemma, basic for our development.

LEMMA 2.1. In the above situation $J_{1} E_{1} E_{2}=E_{1} J_{2} E_{1}=E_{2} J_{1} E_{1}$.

Proof. $J_{1} E_{1} E_{2}=\mathbf{J} E_{1} E_{2}=E_{1} \mathbf{J} E_{2}=E_{1} J_{2} E_{2}$ and symmetrically.

We recall also [3] for the following

LEMMA 2.2. In the above situation let $R_{1}\left(R_{2}\right)$ be the von Neumann subalgebra of $M_{1}$ (resp. $\left.M_{2}\right)$ generated by the range of $\lambda_{2,1}$ (resp. $\left.\lambda_{1,2}\right)$. Then there are norm one projections $\varepsilon_{i}$ from $M_{i}$ to $R_{i}$ which preserve $\left(\omega_{\Omega}\right)_{i}(i=1,2)$.

Proof. Cf. Lemma 5.1, [3].

3. Markovianity on triples of mutually commuting von Neumann algebras. In this section we consider a triple $M_{1}, M_{2}, M_{3}$ of mutually commuting von Neumann 
algebras which act on a Hilbert space $H$ containing a vector $\Omega$ separating for the von Neumann algebra $M$ generated by $M_{1} \cup M_{2} \cup M_{3}$. For $i, j=1,2,3, i \neq j$, we denote by $M_{\{i, j\}}$ the von Neumann algebra generated by $M_{i} \cup M_{j}$, and endow with the index $\{i, j\}$ all the already introduced objects when referred to $M_{\{i, j\}}$. If $k=1,2,3, k \neq i, j$, then $M_{\{i, j\}}$ commutes with $M_{k}$. We shall generalize this notation in the natural way when dealing with more than three mutually commuting von Neumann algebras.

THEOREM 3.1. The following statements are equivalent:

a. $\lambda_{3,\{1,2\}}\left(M_{3}\right)$ is contained in $M_{2}$.

b. $\lambda_{3,\{1,2\}}=\lambda_{3,2}$.

c. $E_{\{1,2\}} E_{3}=E_{2} E_{3}$.

d. $\lambda_{3,\{1,2\}}\left(M_{3}\right)$ is contained in $F\left(\varepsilon_{\{1,2\}, 2}\right)$.

Proof. In the following we take $a_{3}$ in $M_{3}$, and use the fact that by Lemma 2.1, $J_{2} E_{2} a_{3} \Omega=E_{2} J_{3} a_{3} \Omega$ and $E_{\{1,2\}} J_{3} a_{3} \Omega=J_{\{1,2\}} E_{\{1,2\}} a_{3} \Omega$.

a. $\Rightarrow$ b.

$$
\begin{aligned}
& \lambda_{3,\{1,2\}}\left(a_{3}\right) \Omega=E_{2} \lambda_{3,\{1,2\}}\left(a_{3}\right) \Omega \\
& =E_{2} J_{\{1,2\}} E_{\{1,2\}} a_{3}^{+} \Omega=E_{2} E_{\{1,2\}} J_{3} a_{3}^{+} \Omega \\
& =E_{2} J_{3} a_{3}^{+} \Omega=J_{2} E_{2} a_{3}^{+} \Omega=\lambda_{3,2}\left(a_{3}\right) \Omega,
\end{aligned}
$$

which is b. since $\Omega$ is separating for $M_{1,2}$.

b. $\Rightarrow$ c.

$$
\begin{aligned}
& E_{\{1,2\}} J_{3} a_{3} \Omega=J_{\{1,2\}} E_{\{1,2\}} a_{3} \Omega \\
& =\lambda_{3,\{1,2\}}\left(a_{3}^{+}\right) \Omega=\lambda_{3,2}\left(a_{3}^{+}\right) \Omega \\
& =J_{2} E_{2} a_{3} \Omega=E_{2} J_{3} a_{3} \Omega
\end{aligned}
$$

which implies c.

c. $\Rightarrow$ d.

$$
\begin{aligned}
& \varepsilon_{\{1,2\}}\left(\lambda_{3,\{1,2\}}\left(a_{3}\right)\right) \Omega=J_{2} E_{2} a_{3}^{+} \Omega \\
& =E_{2} J_{3} a_{3}^{+} \Omega=E_{\{1,2\}} J_{3} a_{3}^{+} \Omega \\
& =J_{\{1,2\}} E_{\{1,2\}} a_{3}^{+} \Omega=\lambda_{2,\{1,2\}}\left(a_{3}\right) \Omega ;
\end{aligned}
$$

so $\varepsilon_{\{1,2\}, 2}\left(\lambda_{3,\{1,2\}}\left(a_{3}\right)\right)=\lambda_{3,\{1,2\}}\left(a_{3}\right)$.

d. $\Rightarrow$ a. Trivial.

Definition 3.2. We shall say $\Omega$ to be a Markov vector (and $\omega_{\Omega}$ to be a Markov state) for $M$ with respect to the localization $\left(M_{1}, M_{2}, M_{3}\right)$ if the equivalent conditions of Theorem 3.1 are met.

Since in the abelian case $\lambda_{3,\{1,2\}}$ is nothing else than the restriction to $M_{3}$ of the $\left(\omega_{\Omega}\right)_{M}$ preserving conditional expectation from $M$ to $M_{\{1,2\}}$ by a. in Theorem 3.1 our definition is a generalization of the classical notion of markovianity.

THEOREM 3.3. The state $\omega_{\Omega}$ is a Markov state with respect to the localization $\left(M_{1}, M_{2}\right.$, $\left.M_{3}\right)$ iff for all $a_{1} \in M_{1}, a_{3} \in M_{3}$ we have

$$
\lambda_{\{1,3\}, 2}\left(a_{1} a_{3}\right)=\lambda_{1,2}\left(a_{1}\right) \lambda_{3,2}\left(a_{3}\right) .
$$

If so then $\lambda_{1,2}\left(M_{1}\right)$ commutes with $\lambda_{3,2}\left(M_{3}\right)$. 
Pro of. We have:

$$
\begin{aligned}
& \lambda_{\{1,3\}, 2}\left(a_{1} a_{3}\right) \Omega=J_{2} E_{2} a_{3}^{+} a_{1}^{+} \Omega=j_{2} E_{2} a_{1}^{+} a_{3}^{+} \Omega \\
& =J_{2} E_{2} E_{\{1,2\}} a_{1}^{+} a_{3}^{+} \Omega=J_{2} E_{2} a_{1}^{+} E_{\{1,2\}} a_{3}^{+} \Omega \\
& =J_{2} E_{2} a_{1}^{+} J_{\{1,2\}} \lambda_{3,\{1,2\}}\left(a_{3}\right) \Omega .
\end{aligned}
$$

On the other hand:

$$
\lambda_{1,2}\left(a_{1}\right) \lambda_{3,2}\left(a_{3}\right) \Omega=J_{2} E_{2} a_{1}^{+} J_{2} \lambda_{3,2}\left(a_{3}\right) \Omega .
$$

If $\Omega$ is markovian then by c. in Theorem 3.1:

$$
\begin{aligned}
& J_{\{1,2\}} \lambda_{3,\{1,2\}}\left(a_{3}\right) \Omega=E_{\{1,2\}} a_{3}^{+} \Omega \\
& =E_{2} a_{3}^{+} \Omega=J_{2} \lambda_{3,2}\left(a_{3}\right) \Omega
\end{aligned}
$$

and $(*)$ follows.

Conversely, $(*)$ implies for all $a_{i} \in M_{i}(i=1,2,3)$ :

$$
\begin{aligned}
& \left\langle a_{1} a_{2} \Omega, J_{\{1,2\}} \lambda_{3,\{1,2\}}\left(a_{3}\right) \Omega\right\rangle=\left\langle a_{2} \Omega, E_{2} a_{1}^{+} J_{\{1,2\}} \lambda_{3,\{1,2\}}\left(a_{3}\right) \Omega\right\rangle \\
& =\left\langle a_{2} \Omega, E_{2} a_{1}^{+} J_{2} \lambda_{3,2}\left(a_{3}\right) \Omega\right\rangle=\left\langle a_{1} a_{2} \Omega, J_{2} \lambda_{3,2}\left(a_{3}\right) \Omega\right\rangle .
\end{aligned}
$$

As both $J_{\{1,2\}} \lambda_{3,\{1,2\}}\left(a_{3}\right) \Omega$ and $J_{2} \lambda_{3,2}\left(a_{3}\right) \Omega$ are in $H_{\{1,2\}}$ we get:

$$
E_{\{1,2\}} a_{3}^{+} \Omega=J_{\{1,2\}} \lambda_{3,\{1,2\}}\left(a_{3}\right) \Omega=J_{2} \lambda_{3,2}\left(a_{3}\right) \Omega=E_{2} a_{3}^{+} \Omega,
$$

which is c. in Theorem 3.1 .

Let $(*)$ be satisfied. Then

$$
\begin{aligned}
& \lambda_{1,2}\left(a_{1}\right) \lambda_{3,2}\left(a_{3}\right)=\lambda_{\{1,3\}, 2}\left(a_{1} a_{3}\right)=\lambda_{\{1,3\}, 2}\left(a_{3} a_{1}\right) \\
& =\lambda_{\{1,3\}, 2}\left(\left(a_{1}^{+} a_{3}^{+}\right)^{+}\right)=\left(\lambda_{1,2}\left(a_{1}^{+}\right) \lambda_{3,2}\left(a_{3}^{+}\right)\right)^{+}=\lambda_{3,2}\left(a_{3}\right) \lambda_{1,2}\left(a_{1}\right) .
\end{aligned}
$$

COROLlary 3.4 (reversibility). The state $\omega_{\Omega}$ is a Markov state for $M$ with respect to the localization $\left(M_{1}, M_{2}, M_{3}\right)$ iff it is a Markov state for $M$ with respect to the localization $\left(M_{3}, M_{2}, M_{1}\right)$.

Proof. If $\Omega$ is Markovian for $M$ with respect to the localization $\left(M_{1}, M_{2}, M_{3}\right)$ then by Theorem 3.3

$$
\begin{aligned}
& \lambda_{\{3,1\}, 2}\left(a_{3} a_{1}\right)=\lambda_{\{1,3\}, 2}\left(a_{1} a_{3}\right) \\
& =\lambda_{1,2}\left(a_{1}\right) \lambda_{3,2}\left(a_{3}\right)=\lambda_{3,2}\left(a_{3}\right) \lambda_{1,2}\left(a_{1}\right) .
\end{aligned}
$$

Now by the converse implication of Theorem 3.3 our claim follows.

\section{Noncommutative Markov chains}

Lemma 4.1. Let $M_{i}(i=1, \ldots, n ; n \geq 4)$ be mutually commuting von Neumann algebras acting on a Hilbert space $H$ and $\Omega$ be markovian for $M_{\{1, \ldots, k\}}$ with respect to the localization $\left(M_{\{1, \ldots, k-2\}}, M_{k-1}, M_{k}\right)$ for all $k=3, \ldots, n$. Then for all $a_{k} \in M_{k}(k=$ $3, \ldots, n)$ we have:

$$
\begin{aligned}
& \lambda_{\{3,4, \ldots, n\},\{1,2\}}\left(a_{3} a_{4} \ldots a_{n}\right) \Omega \\
& =E_{2} J_{3} a_{3}^{+} J_{3} E_{3} \ldots E_{n-2} J_{n-1} a_{n-1}^{+} J_{n-1} \lambda_{n, n-1}\left(a_{n}\right) \Omega .
\end{aligned}
$$


Pr o of. By induction. For $n=3$ our equality is the Markov property of $\Omega$ for $M_{\{1,2,3\}}$ with respect to the localization $\left(M_{1}, M_{2}, M_{3}\right)$. On the other hand by the induction hypothesis applied to $\lambda_{\{4, \ldots, n\},\{1,2,3\}}\left(a_{3} a_{4} \ldots a_{n}\right) \Omega$ we get:

$$
\begin{aligned}
& \lambda_{\{3,4, \ldots, n\},\{1,2\}}\left(a_{3} a_{4} \ldots a_{n}\right) \Omega=J_{\{1,2\}} E_{\{1,2\}} a_{3}^{+} a_{4}^{+} \ldots a_{n}^{+} \Omega \\
& =J_{\{1,2\}} E_{\{1,2\}} E_{\{1,2,3\}} a_{3}^{+} a_{4}^{+} \ldots a_{n}^{+} \Omega \\
& =J_{\{1,2\}} E_{\{1,2\}} a_{3}^{+} E_{\{1,2,3\}} a_{4}^{+} \ldots a_{n}^{+} \Omega \\
& =J_{\{1,2\}} E_{\{1,2\}} a_{3}^{+} J_{\{1,2,3\}} \lambda_{\{4, \ldots, n\},\{1,2,3\}}\left(a_{4} \ldots a_{n}\right) \Omega \\
& =J_{\{1,2\}} E_{\{1,2\}} a_{3}^{+} J_{\{1,2,3\}} E_{3} J_{4} a_{4}^{+} J_{4} E_{4} \ldots E_{n-2} J_{n-1} a_{n-1}^{+} J_{n-1} \lambda_{n, n-1}\left(a_{n}\right) \Omega \\
& =J_{\{1,2\}} E_{\{1,2\}} a_{3}^{+} E_{3} a_{4}^{+} J_{4} E_{4} \ldots E_{n-2} J_{n-1} a_{n-1}^{+} J_{n-1} \lambda_{n, n-1}\left(a_{n}\right) \Omega \\
& =E_{\{1,2\}} J_{3} a_{3}^{+} E_{3} a_{4}^{+} J_{4} E_{4} \ldots E_{n-2} J_{n-1} a_{n-1}^{+} J_{n-1} \lambda_{n, n-1}\left(a_{n}\right) \Omega \\
& =E_{2} J_{3} a_{3}^{+} J_{3} E_{3} J_{4} a_{4}^{+} J_{4} E_{4} \ldots E_{n-2} J_{n-1} a_{n-1}^{+} J_{n-1} \lambda_{n, n-1}\left(a_{n}\right) \Omega .
\end{aligned}
$$

Proposition 4.2. Let the hypothesis of Lemma 4.1 be satisfied. Then $\Omega$ is markovian for $M_{\{1, \ldots, n\}}$ with respect to the localization $\left.M_{\{1, \ldots, k-1\}}, M_{k}, M_{\{k+1, \ldots, n\}}\right)$ for $k=$ $2, \ldots, n-1$. Moreover for $j=1, \ldots n-3, a_{n} \in M_{n}$ we have:

$$
\lambda_{n-j, n-j-1}\left(\ldots\left(\lambda_{n-1, n-2}\left(\lambda_{n, n-1}\left(a_{n}\right)\right)\right) \ldots\right)=\lambda_{n, n-j-1}\left(a_{n}\right),
$$

when $j$ is even (chain rule) and

$$
\lambda_{n-j, n-j-1}\left(\ldots\left(\lambda_{n-1, n-2}\left(\lambda_{n, n-1}\left(a_{n}\right)\right)\right) \ldots\right) \Omega=J_{n-j-1} \lambda_{n, n-j-1}\left(a_{n}^{+}\right) \Omega
$$

when $j$ is odd

Proof. By Lemma 4.1 we have for $a_{k} \in M_{k}(k=3, \ldots, n)$ :

$$
\begin{aligned}
& E_{\{1,2\}} a_{3} a_{4} \ldots a_{n} \Omega=J_{\{1,2\}} \lambda_{\{3,4, \ldots, n\},\{1,2\}}\left(a_{3}^{+} a_{4}^{+} \ldots a_{n}^{+}\right) \Omega \\
& =J_{\{1,2\}} E_{2} J_{3} a_{3} J_{3} E_{3} \ldots E_{n-2} J_{n-1} a_{n-1} J_{n-1} \lambda_{n, n-1}\left(a_{n}\right) \Omega \\
& =J_{\{1,2\}} E_{\{1,2\}} J_{3} a_{3} J_{3} E_{3} \ldots E_{n-2} J_{n-1} a_{n-1} J_{n-1} \lambda_{n, n-1}\left(a_{n}\right) \Omega \\
& =E_{\{1,2\}} a_{3} J_{3} E_{3} \ldots E_{n-2} J_{n-1} a_{n-1} J_{n-1} \lambda_{n, n-1}\left(a_{n}\right) \Omega \\
& =J_{2} E_{2} J_{3} a_{3} J_{3} E_{3} \ldots E_{n-2} J_{n-1} a_{n-1} J_{n-1} \lambda_{n, n-1}\left(a_{n}\right) \Omega \\
& =J_{2} \lambda_{\{3,4, \ldots, n\},\{1,2\}}\left(a_{3}^{+} a_{4}^{+} \ldots a_{n}^{+}\right) \Omega=E_{2} a_{3} a_{4} \ldots a_{n} \Omega ;
\end{aligned}
$$

so c. in Theorem 3.1 is satisfied for $\Omega$ and $M_{\{1, \ldots, n\}}$ with respect to the localization $\left(M_{1}, M_{2}, M_{\{3, \ldots, n\}}\right)$.

Our hypothesis now allows us to let $M_{\{1, \ldots, k-1\}}$ play the role of $M_{1}$ above, $M_{k}$ the role of $M_{2}$ and $M_{\{k+1, \ldots, n\}}$ of $M_{\{3, \ldots, n\}}$ and we get our first claim.

Let us now prove our second claim for $j$ even. Then, applying Lemma 4.1 to the localization $\left(M_{\{1, \ldots, n-j-2\}}, M_{n-j-1}, M_{\{n-j, \ldots, n\}}\right)$ and setting $a_{n-1}=a_{n-2}=\ldots=a_{n-j}=l$, we get:

$$
\begin{aligned}
& \lambda_{n, n-j-1}\left(a_{n}\right) \Omega=\lambda_{\{n-j, \ldots, n\},\{1, \ldots, n-j-1\}}\left(a_{n}\right) \Omega \\
& =E_{n-j-1} E_{n-j} \ldots E_{n-2} \lambda_{n, n-1}\left(a_{n}\right) \Omega \\
& =J_{n-j-1} E_{n-j-1} J_{n-j} E_{n-j} J_{n-j+1} \ldots j_{n-3} E_{n-3} J_{n-2} E_{n-2} \lambda_{n, n-1}\left(a_{n}\right) \Omega \\
& =\lambda_{n-j, n-j-1}\left(\ldots\left(\lambda_{n-1, n-2}\left(\lambda_{n, n-1}\left(a_{n}\right)^{+}\right)^{+}\right) \ldots{ }^{+}\right) \Omega,
\end{aligned}
$$

and our claim follows since $\Omega$ is separating for $M_{n-j-1}$. 
If $j$ is odd we have, using the preceding case:

$$
\begin{aligned}
& \lambda_{n, n-j-1}\left(a_{n}\right) \Omega \\
& =E_{n-j-1} J_{n-j} E_{n-j} J_{n-j+1} \ldots J_{n-3} E_{n-3} J_{n-2} E_{n-2} \lambda_{n, n-1}\left(a_{n}\right) \Omega \\
& =E_{n-j-1} \lambda+n-j+1, n-j\left(\ldots\left(\lambda_{n-1, n-2}\left(\lambda_{n, n-1}\left(a_{n}\right)^{+}\right)^{+}\right) \ldots+\right) \Omega \\
& =J_{n-j-1} \lambda_{n-j, n-j-1}\left(\ldots\left(\lambda_{n-1, n-2}\left(\lambda_{n, n-1}\left(a_{n}\right)\right)\right) \ldots\right) \Omega .
\end{aligned}
$$

Proposition 4.3. Let $M_{i}$ (i integer) be the von Neumann algebras acting on a Hilbert space $H$. We call $M_{[i}(M)$ the von Neumann algebra generated by the union of $M_{k}$ with $k \geq i$ (by the union of all $M_{k}$ ). We assume the vector $\Omega$ in $H$ to be markovian for $M_{\{1, \ldots, k\}}$ with respect to the localization $\left(M_{\{1, \ldots, k-2\}}, M_{k-1}, M_{k}\right)$ for all integers $k$. Then for all integers $k \Omega$ is markovian for $M$ with respect to the localization $\left(M_{\{1, \ldots, k-2\}}, M_{k-1}, M_{[k}\right)$.

Proof. The projection $E_{[k}$ on $H$ is the supremum of the projections $E_{\{k, \ldots, k+n\}}$ for $n$ natural. So by Proposition 4.2 we have (the limit is taken in the strong operator topology):

$$
\begin{aligned}
& E_{\{1, \ldots, k-1\}} E_{[k}=\lim E_{\{1, \ldots, k-1\}} E_{\{k, \ldots, k+n\}} \\
& =\lim E_{k-1} E_{\{k, \ldots, k+n\}}=E_{k-1} E_{[k},
\end{aligned}
$$

and c. in Theorem 3.1 is proved for our localization.

THEOREM 4.4. Let for all natural numbers $i M_{i}$ be a von Neumann algebra acting on a Hilbert space $H$ and $\Omega$ in $H$ be Markovian for $M_{\{1, \ldots, k\}}$ with respect to the localization $\left(M_{\{1, \ldots, k-2\}}, M_{k-1}, M_{k}\right)$ for $k$ natural. Let $A, B, C$ be subsets of the natural numbers such that for $a$ in $A, b$ in $B, c$ in $C$ we have $a<b<c$. Then $\Omega$ is Markovian for $M_{A \cup B \cup C}$ with respect to the localization $\left(M_{A}, M_{B}, M_{C}\right)$.

Proof. Let $b=\max B$, By prop. 4.3 for all $a_{C}$ in $M_{C}$ we have $E_{A \cup B} a_{C} \Omega=E_{b} a_{C} \Omega$, which implies $E_{A \cup B} a_{c} \Omega=E_{B} a_{c} \Omega$ and c. in th. 3.1 is satisfied for our localization.

\section{A structure theorem for markovian states}

TheOREM 5.1. Let $\Omega$ be a Markov state for $M$ with respect to the localization $\left(M_{1}, M_{2}\right.$, $\left.M_{3}\right)$. We set $M_{2,3}\left(M_{2,1}, M_{1,2}, M_{3,2}\right)$ to be the von Neumann subalgebra of $M_{2}$ generated by the range of $\lambda_{3,2}$ (resp. of $\left.\lambda_{1,2}, \lambda_{2,1}, \lambda_{2,3}\right), N_{1}\left(N_{2}, N_{3}, N\right)$ the von Neumann algebra generated by $M_{2,1} \cup M_{1,2}$ (resp. $M_{2,1} \cup M_{2,3}, M_{2,3} \cup M_{3,2}, M_{2,1} \cup M_{1,2} \cup M_{2,3} \cup M_{3,2}$ ).

Then $N_{1}$ and $N_{3}$ mutually commute and there are $\omega_{\Omega}$ preserving norm one projections $\varepsilon: M \mapsto N, \varepsilon_{1}: M_{1} \mapsto M_{1,2}, \varepsilon_{2}: M_{2} \mapsto N_{2}$ and $\varepsilon_{3}: M_{3} \mapsto M_{3,2}$ such that for all $a_{i}$ in $M_{i}(i=1,2,3)$

$$
\varepsilon\left(a_{1} a_{2} a_{3}\right)=\varepsilon_{1}\left(a_{1}\right) \varepsilon_{2}\left(a_{2}\right) \varepsilon_{3}\left(a_{3}\right) .
$$

Further let us denote by $\lambda^{N_{3,1}}\left(\lambda^{N_{1,3}}\right)$ the dual of the $\Omega$ implemented odd stochastic coupling for $N_{3}$ and $N_{1}$ (resp. for $N_{1}$ and $N_{3}$ ), and by $Z_{2,1}\left(Z_{2,3}\right)$ the center of $M_{2,1}\left(M_{2,3}\right)$. Then $\lambda^{N_{3,1}}\left(N_{3}\right) \subseteq Z_{2,1}$ and $\lambda^{N_{1,3}}\left(N_{1}\right) \subseteq Z_{2,3}$.

Pro of. By Theorem $3.3 M_{2,3}$ and $M_{2,1}$ mutually commute; this implies that $N_{1}$ and $N_{3}$ also mutually commute. We note also that Lemma 2.2 gives the existence of $\varepsilon_{1}$ and $\varepsilon_{3}$ as above, as well as the existence of $\omega_{\Omega}$ preserving norm one projections $\varepsilon_{2,1}$ and $\varepsilon_{2,3}$ from $M_{2}$ to $M_{2,1}$ and to $M_{2,3}$. This implies the existence of $\varepsilon_{2}$. 
We have now, if $b_{1} \in M_{1,2}, b_{2} \in N_{2}$ and $b_{3} \in M_{3,2}$ :

$$
\begin{aligned}
& \left\langle b_{1} b_{2} b_{3} \Omega, a_{1} a_{2} a_{3} \Omega\right\rangle=\left\langle a_{1}^{+} a_{2}^{+} b_{1} b_{2} \Omega, b_{3}^{+} a_{3} \Omega\right\rangle \\
& =\left\langle J_{3} \lambda_{\{1,2\}, 3}\left(a_{1}^{+} a_{2}^{+} b_{1} b_{2}\right) \Omega, b_{3}^{+} a_{3} \Omega\right\rangle \\
& =\left\langle J_{3} \lambda_{\{1,2\}, 3}\left(a_{1}^{+} a_{2}^{+} b_{1} b_{2}\right) \Omega, \varepsilon_{3}\left(b_{3}^{+} a_{3}\right) \Omega\right\rangle \\
& =\left\langle J_{3} \lambda_{\{1,2\}, 3}\left(a_{1}^{+} a_{2}^{+} b_{1} b_{2}\right) \Omega, b_{3}^{+} \varepsilon_{3}\left(a_{3}\right) \Omega\right\rangle \\
& =\left\langle a_{2}^{+} \varepsilon_{3}\left(a_{3}\right)^{+} b_{2} b_{3} \Omega, b_{1}^{+} a_{1} \Omega\right\rangle=\left\langle a_{2}^{+} \varepsilon_{3}\left(a_{3}\right)^{+} b_{2} b_{3} \Omega, b_{1}^{+} \varepsilon_{1}\left(a_{1}\right) \Omega\right\rangle \\
& =\left\langle\varepsilon_{1}\left(a_{1}\right)^{+} b_{1} \varepsilon_{3}\left(a_{3}\right)^{+} b_{3} \Omega, b_{2}^{+} a_{2} \Omega\right\rangle \\
& =\left\langle J_{2} \lambda_{\{1,3\}, 2}\left(b_{1}^{+} \varepsilon_{1}\left(a_{1}\right) b_{3}^{+} \varepsilon_{3}\left(a_{3}\right)^{+}\right) \Omega, b_{2}^{+} a_{2} \Omega\right\rangle \\
& =\left\langle J_{2} \lambda_{1,2}\left(b_{1}^{+} \varepsilon_{1}\left(a_{1}\right)\right) \lambda_{3,2}\left(b_{3}^{+} \varepsilon_{3}\left(a_{3}\right)^{+}\right) \Omega, b_{2}^{+} a_{2} \Omega\right\rangle \\
& =\left\langle J_{2} \lambda_{1,2}\left(b_{1}^{+} \varepsilon_{1}\left(a_{1}\right)\right) \lambda_{3,2}\left(b_{3}^{+} \varepsilon_{3}\left(a_{3}\right)^{+}\right) \Omega, \varepsilon_{2}\left(b_{2}^{+} a_{2}\right) \Omega\right\rangle \\
& =\left\langle J_{2} \lambda_{1,2}\left(b_{1}^{+} \varepsilon_{1}\left(a_{1}\right)\right) \lambda_{3,2}\left(b_{3}^{+} \varepsilon_{3}\left(a_{3}\right)^{+}\right) \Omega, b_{2}^{+} \varepsilon_{2}\left(a_{2}\right) \Omega\right\rangle \\
& =\left\langle\varepsilon_{1}\left(a_{1}\right)^{+} b_{1} \varepsilon_{3}\left(a_{3}\right)^{+} b_{3} \Omega, b_{2}^{+} \varepsilon_{2}\left(a_{2}\right) \Omega\right\rangle \\
& =\left\langle b_{1} b_{2} b_{3} \Omega, \varepsilon_{1}\left(a_{1}\right) \varepsilon_{2}\left(a_{2}\right) \varepsilon_{3}\left(a_{3}\right) \Omega\right\rangle,
\end{aligned}
$$

so our first claim follows.

The vector $\Omega$ is obviously markovian with respect to the localization $\left(N_{1}, M_{2,3}, M_{3,2}\right)$ for the von Neumann algebra $N$; it is also markovian with respect to the localization $\left(M_{1,2}, M_{2,1}, M_{2,3}\right)$ for the von Neumann algebra generated by the union of these latter algebras (it is obvious that the von Neumann algebras involved in the above triples mutually commute). It follows then by Proposition 4.2 that it is markovian with respect to the localization $\left(M_{1,2}, M_{2,1}, N_{3}\right)$ for the von Neumann algebra generated by their union. This implies by Theorem 3.1 a. the range of $\lambda^{N_{3,1}}$ to be contained in $M_{2,1}$. We also note that the dual of the $\Omega$ implemented odd stochastic transition for $M_{1,2}$ and $M_{2,1}$ coincides with the restriction of $\lambda_{1,2}$ to $M_{1,2}$ and that its range generates $M_{2,1}$ By the first part of this theorem the ranges of $\lambda^{N_{3,1}}$ and of this latter mapping commute; $\lambda^{N_{3,1}}\left(N_{3}\right) \subseteq Z_{2,1}$. Symmetrically we prove that $\lambda^{N_{1,3}}\left(N_{1}\right) \subseteq Z_{2,3}$.

EXAMPLE 5.2. Let us assume in Theorem $5.1 \lambda_{1,2}$ and $\lambda_{3,2}$ to be surjective, $M_{2,1}$ and $M_{2,3}$ to be factors and $M_{2}$ to be generated by their union. Then Theorem 5.1 implies that $\left(\omega_{\Omega}\right)_{M}$ is a state product of its restrictions to the von Neumann subalgebras generated by the union of $M_{1}$ and $M_{2,1}$ and of $M_{2,3}$ and $M_{3}$.

THEOREM 5.3. Let $\Omega$ be a Markov state for $M$ with respect to the localization $\left(M_{1}\right.$, $\left.M_{2}, M_{3}\right)$ and $\sigma_{t}$ be the modular authomorphism group for $\left(\omega_{\Omega}\right)_{M}$ on $M$. Then $\sigma_{t}\left(M_{1}\right) \subseteq$ $M_{\{1,2\}}$ for all real $t$.

Pro of. We shall use the notations established in Theorem 5.1 and prove that the von Neumann algebra $L_{1}$ generated by the union of $M_{1}$ and $M_{2,1}$ is $\left(\omega_{\Omega}\right)_{M}$ expected in $M$. This will imply $\sigma_{t}\left(M_{1}\right) \subseteq L_{1}$ and therefore our claim.

Let $L_{3}$ be the von Neumann algebra generated by $M_{3} \cup M_{2,3}$, and $L$ the von Neumann algebra generated by $L_{1} \cup L_{3}$. We prove first that $L$ is $\left(\omega_{\Omega}\right)_{M}$ expected in $M$. Let $a_{1}, b_{1} \in$ $M_{1}, a_{3}, b_{3} \in M_{3}, a_{2} \in M_{2}$ and $b_{2} \in N_{2}$. Then: 


$$
\begin{aligned}
& \left\langle b_{1} b_{2} b_{3} \Omega, a_{1} a_{2} a_{3} \Omega\right\rangle=\left\langle a_{1}^{+} b_{1} a_{3}^{+} b_{3} \Omega, b_{2}^{+} a_{2} \Omega\right\rangle \\
& =\left\langle J_{2} \lambda_{\{1,3\}, 2}\left(b_{1}^{+} a_{1} b_{3}^{+} a_{3}^{+}\right) \Omega, b_{2}^{+} a_{2} \Omega\right\rangle \\
& =\left\langle J_{2} \lambda_{\{1,3\}, 2}\left(b_{1}^{+} a_{1} b_{3}^{+} a_{3}^{+}\right) \Omega, \varepsilon_{2}\left(b_{2}^{+} a_{2}\right) \Omega\right\rangle \\
& =\left\langle J_{2} \lambda_{\{1,3\}, 2}\left(b_{1}^{+} a_{1} b_{3}^{+} a_{3}^{+}\right) \Omega, b_{2}^{+} \varepsilon_{2}\left(a_{2}\right) \Omega\right\rangle \\
& =\left\langle a_{1}^{+} b_{1} a_{3}^{+} b_{3} \Omega, b_{2}^{+} \varepsilon_{2}\left(a_{2}\right) \Omega\right\rangle \\
& =\left\langle b_{1} b_{2} b_{3} \Omega, a_{1} \varepsilon_{2}\left(a_{2}\right) a_{3} \Omega\right\rangle,
\end{aligned}
$$

so the required projection $\varepsilon_{L}$ is obtained by setting

$$
\varepsilon_{L}\left(a_{1} a_{2} a_{3}\right)=a_{1} \varepsilon_{2}\left(a_{2}\right) a_{3}
$$

and extending it then by linearity and continuity to $M$.

Let $\lambda^{L_{3,1}}$ be the dual of the stochastic coupling for $\left(L_{3}, L_{1}\right)$ implemented by $\Omega$. Then $\lambda^{L_{3,1}}\left(L_{3}\right) \subseteq Z_{2,1}$. As $M_{2,3} \supseteq \lambda_{3,\{1,2\}}\left(M_{\{1,2\}}\right), \Omega$ is markovian on $L$ with respect to the localization $\left(L_{1}, M_{2,3}, M_{3}\right), \Omega$ is also markovian on the von Neumann algebra generated by the union of $L_{1}$ and $M_{2,3}$ with respect to the localization $\left(L_{1}, Z_{2,1}, M_{2,3}\right)$. Indeed if we take $a_{1}$ in $M_{1}, a_{2,1}$ in $M_{2,1}$ and $a_{2,3}$ in $M_{2,3}$, and remember that $E_{2,3} E_{2,1}=E^{Z_{2,3}} E_{2,1}$; this follows from:

$$
\begin{aligned}
& \left\langle a_{1} a_{2,1} \Omega, a_{2,3} \Omega\right\rangle=\left\langle a_{2,1} E_{2,1} a_{1} \Omega, a_{2,3} \Omega\right\rangle \\
& =\left\langle E_{2,1} a_{2,1} a_{1} \Omega, a_{2,3} \Omega\right\rangle=\left\langle E^{Z_{2,3}} E_{2,1} a_{2,1} a_{1} \Omega, a_{2,3} \Omega\right\rangle \\
& =\left\langle E_{2,1} a_{2,1} a_{1} \Omega, E^{Z_{2,3}} a_{2,3} \Omega\right\rangle=\left\langle E^{Z_{2,1}} a_{2,1} a_{1} \Omega, a_{2,3} \Omega\right\rangle \\
& =\left\langle a_{2,1} a_{1} \Omega, E^{Z_{2,1}} a_{2,3} \Omega\right\rangle .
\end{aligned}
$$

Now Lemma 4.1 yields that $\Omega$ is markovian on $L$ for the localization $\left(L_{1}, Z_{1,2}, M_{2,3}\right)$, which implies $\lambda^{L_{3,1}}\left(L_{3}\right)$ to be contained in $Z_{2,1}$.

Let now $a_{1}$ be in $L_{1}, a_{3}$ in $L_{3}$ and $E^{L_{1}}$ the projection on the closure of $\left\{a_{1} \Omega: a_{1} \in L_{1}\right\}$. We have:

$$
\begin{aligned}
& E^{L_{1}} a_{1} a_{3} \Omega=a_{1} E^{L_{1}} a_{3} \Omega \\
& =a_{1} J_{1} \lambda^{L_{3,1}}\left(a_{3}^{+}\right) \Omega=a_{1} \lambda^{N_{3,1}}\left(a_{3}\right) \Omega,
\end{aligned}
$$

and the mapping $\varepsilon^{L_{1}}$ obtained by setting $\varepsilon^{L_{1}}\left(a_{1} a_{3}\right)=a_{1} \lambda^{N_{3,1}}\left(a_{3}\right)$ and extending it once more to $L_{1}$ by linearity and continuity is the required $\left(\omega_{\Omega}\right)_{L}$ preserving generalized conditional expectation from $L$ to $L_{1}$. This completes the proof.

\section{References}

[1] L. Accardi and C. Cecchini, Conditional expectations in von Neumann algebras and a theorem of Takesaki, J. Funct. Anal. 45 (1982), 245-273.

[2] C. Cecchini, On the structure of quantum Markov processes, Quantum Probability and Related Topics Vol. IX, 149-157, World Scientific.

[3] C. Cecchini, Stochastic coupling for von Neumann algebras, preprint. 